\title{
A low power multiplexer based pass transistor logic full adder
}

\begin{abstract}
In this paper, a high-speed low-power full adder design using multiplexer based pass transistor logic featuring full-swing output is proposed. The adder is designed and simulated using the industry standard $130 \mathrm{~nm}$ CMOS technology, at a supply voltage of $1.2 \mathrm{~V}$. The obtained Power Delay Product (PDP) of its critical path is $29 \times 10-18 \mathrm{~J}$ and its power consumption is $2.01 \varepsilon \mathrm{W}$. The proposed full adder is also capable to function at lower supply voltages of $0.4 \mathrm{~V}$ and $0.8 \mathrm{~V}$ without significant performance degradation. The proposed adder when cascaded in a 4-bit ripple carry adder configuration, its power, delay and PDP performance are better than the other adders making it suitable for larger arithmetic circuits.
\end{abstract}

Keyword: Delay; Full adder; Low power; Multiplexer; Pass transistor logic; Power delay product (PDP) 